\title{
Scintigraphic measurement of ileocaecal transit in irritable bowel syndrome and chronic idiopathic constipation
}

\author{
R Hutchinson, A Notghi, N B Smith, L K Harding, D Kumar
}

\begin{abstract}
This study investigated the hypothesis that some features of functional gastrointestinal disorders may be associated with abnormalities of ileocaecal transit by measuring ileocaecal transit using a scintigraphic technique in 43 patients with chronic constipation, 20 patients with irritable bowel syndrome (IBS), and 18 control subjects. Subjects ingested enteric coated capsules, which delivered 111indium radionuclide to the distal ileum. Gammacamera images were acquired at hourly intervals until caecal filling was complete. Ileocaecal transit was defined as the time between peak scintigraphic activity in the terminal ileum and peak activity in the caecum. The mean (SD) ileocaecal transit of 103 (50) minutes in patients with IBS was significantly faster than that in control subjects (mean (SD) ileocaecal transit 174 (78) minutes, $\mathbf{p}<0 \cdot 002)$. There were no significant differences in ileocaecal transit between patients with chronic idiopathic constipation and the control subjects, or between patients with constipation predominant and diarrhoea predominant IBS. This study developed a practical scintigraphic method of measuring ileocaecal transit. The rapid ileocaecal transit in both the constipation and diarrhoea predominant forms of IBS suggests that bloating may not after all result from delayed ileal emptying.

(Gut 1995; 36: 585-589)
\end{abstract}

Keywords: irritable bowel syndrome, ileocaecal transit, constipation, scintigraphy.

\section{Department of Surgery, Queen Elizabeth Hospital, Edgbaston, Birmingham \\ R Hutchinson D Kumar}

Department of Physics and Nuclear Medicine, Dudley Road Hospital, Birmingham

A Notghi

N B Smith

L K Harding

Correspondence to: Mr D Kumar, Department of Surgery, St George's of Surgery, St George's SW17.

Accepted for publication 15 July 1994
Dysfunction of the ileocaecal junction and disturbances of ileocaecal transit have been implicated in causing some of the symptoms of the irritable bowel syndrome (IBS) and other functional bowel disorders. ${ }^{1}$ It has been suggested that patients with constipation predominant IBS have delayed ileocaecal transit, and that those with diarrhoea predominant IBS have accelerated ileocaecal transit. ${ }^{2}$ Trotman and Price studied ileal emptying scintigraphically with radiolabelled bran and found significantly delayed ileal emptying and ileocaecal clearance in women with IBS in whom bloating was the predominant symptom. ${ }^{3}$ They postulated that ileocaecal 'stagnation' may account for the symptoms of IBS. Accelerated small bowel transit in diarrhoea predominant IBS has been shown, but ileocaecal transit was not considered specifically in these studies. ${ }^{45}$ The exact relation between the different forms of IBS and abnormalities of ileocaecal transit remains to be elucidated. Furthermore, it has been suggested that patients with chronic idiopathic constipation may have a generalised disorder of gut motility. ${ }^{67}$ It is reasonable to speculate that ileocaecal transit in these patients may be slow.

The importance of the ileocaecal region in the pathogenesis of functional bowel disorders remains controversial because of difficulties with studying the motility of this comparatively inaccessible region. Manometric studies entail complex and invasive techniques, and the results obtained from laboratory measurements are difficult to extrapolate to the nonlaboratory setting. Measurement of ileocaecal transit is also difficult, and no technique has gained widespread acceptance. The aims of this study were, firstly, to develop a practical scintigraphic method of measuring ileocaecal transit, and secondly, to examine ileocaecal transit in patients with the functional bowel disorders of IBS and chronic idiopathic constipation.

\section{Methods}

PATIENTS

We measured ileocaecal transit in 63 patients with functional bowel disorders and 18 control subjects. All gave full and informed consent. Pregnant women were excluded from the study. The study was approved by the local ethical committee and the Administration of Radioactive Substance Advisory Committee (ARSAC).

The control group consisted of 12 women and six men with a median age of 35 years (range 19-63). Nine subjects had no gastrointestinal symptoms or any history of gastrointestinal disease. Nine subjects in the control group were women who were undergoing investigation for faecal soiling. These subjects all had grade I faecal incontinence and were found to have local anal or pelvic floor conditions causing their symptoms. Median stool frequency was one per day (range 1-3). Mechanical disorders of the colon were excluded by barium enema or colonoscopic examination, and all these subjects had normal colonic transit shown by colonic scintigraphy.

The group with chronic idiopathic constipation consisted of 35 women (median age 42, 
range 19-75) and eight men (median age 43, range 16-58). All these patients fulfilled accepted diagnostic criteria for chronic idiopathic constipation, which was defined as infrequent bowel actions (less than twice per week) or straining at more than $25 \%$ of bowel actions for more than one year, or both. ${ }^{8}$ Mechanical or metabolic causes for symptoms were excluded by haematological tests, gastrointestinal contrast radiology, or colonoscopy, or all three.

There were 20 patients with IBS. The median age was 43 years (range 22-63). All these patients had chronic symptoms and fulfilled accepted diagnostic criteria for IBS. ${ }^{10}$ The median duration of symptoms was five years (range 2-35). As with the chronically constipated patients, mechanical or metabolic causes for symptoms were excluded.

Sixteen female patients (median age 45 , range 22-63) were classified on clinical criteria as having the constipation predominant form of IBS. All these patients complained of constipation in addition to abdominal pain and distension. Their symptoms included ineffective defecation with straining, passage of small amounts of hard stools, or a sensation of incomplete evacuation. All had previously used laxatives or suppositories, or both with no benefit. Two female and two male patients (median age 44, range 39-57) had the diarrhoea predominant form of IBS. These patients complained of faecal urgency and frequent defecation with passage of mucus or loose stools, in addition to their abdominal pain and distension.

\section{STUDY DESIGN}

\section{Ileocaecal scintigraphy}

Subjects received assessment of colonic transit by a scintigraphic technique, which has been described in detail elsewhere. ${ }^{11} 12$ Ileocaecal transit was measured as a routine part of the colonic transit assessment. The protocol entails delivery of a bolus of 111-indium

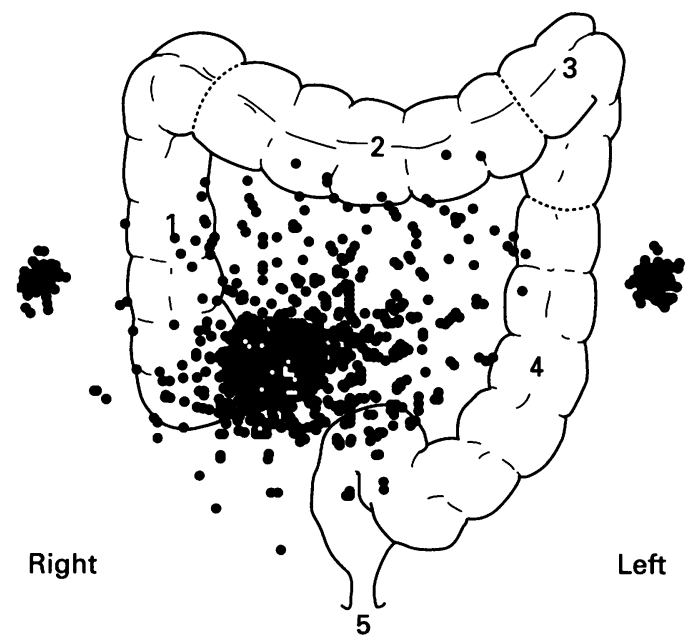

Anterior 09.05 Figure 1: Analogue image acquired after capsule dispersal
showing maximum scintigraphic activity in the vicinity of the distal ileum. radionuclide to the distal ileum in enteric coated capsules. Two MBq of the radionuclide was absorbed onto resin particles and incorporated into gelatin capsules coated with a methacrylate polymer (Eudragit S). ${ }^{12}$ Pilot studies have shown this capsule remains intact on passage through the stomach and proximal small intestine, and there is reliable release of a bolus of radionuclide into the distal ileum. ${ }^{12} 13$

No patients or control subjects were taking drugs likely to affect intestinal transit or defecation at the time of study. Drugs were stopped at least five days before assessment. After an overnight fast, subjects ingested a radiolabelled capsule at 0500 while at home. They attended the nuclear medicine department at 0900. Radioactive (57-cobalt) markers were applied to bony landmarks to permit correct alignment of images. Anterior and posterior gammacamera images were acquired at roughly hourly intervals from 0900 hours until ileal emptying was completed. Subjects were not permitted oral intake until all the radioisotope had passed beyond the ileocaecal junction. Studies in which the radionuclide dispersal occurred prematurely, or in which capsules passed intact into the colon were excluded from this analysis. Image acquisition was continued at less frequent intervals for a further three days for the assessment of colonic transit. Analogue pictures as well as digital computer images were acquired.

\section{Analysis}

Corrections were made for depth of the radioisotopic activity in the abdomen (by calculating a geometric mean from anterior and posterior scans), and for isotope decay. From the computerised data, dynamic images were generated and the position of the colon determined. Figure 1 shows the colonic 'regions of interest'. The counts within each colonic segment were measured on each image. Counts arising from outside the colonic segments were from activity in the small intestine.

We defined ileocaecal transit as the time from peak scintigraphic activity in the distal ileum to peak activity in the caecum. Maximum activity in the caecum was readily identified on analogue images (Fig 2), and this was confirmed by finding maximum counts in the caecal region of interest. There was no difficulty in differentiating caecal from small intestinal activity because the caecum becomes progressively easier to identify as colonic filling progresses.

Maximum activity in the distal ileum was identified in two ways. Firstly, counts from colonic segments were subtracted from the counts of the whole field of view to give the activity in the small intestine. The time of the maximum counts in the small bowel was noted. Secondly, analogue pictures were scrutinised to confirm (a) capsule dispersal in the small bowel and (b) the location of the maximum bolus of activity immediately medial to the region that, on subsequent images, proved to be the caecum. This area was considered to include the distal ileum. 


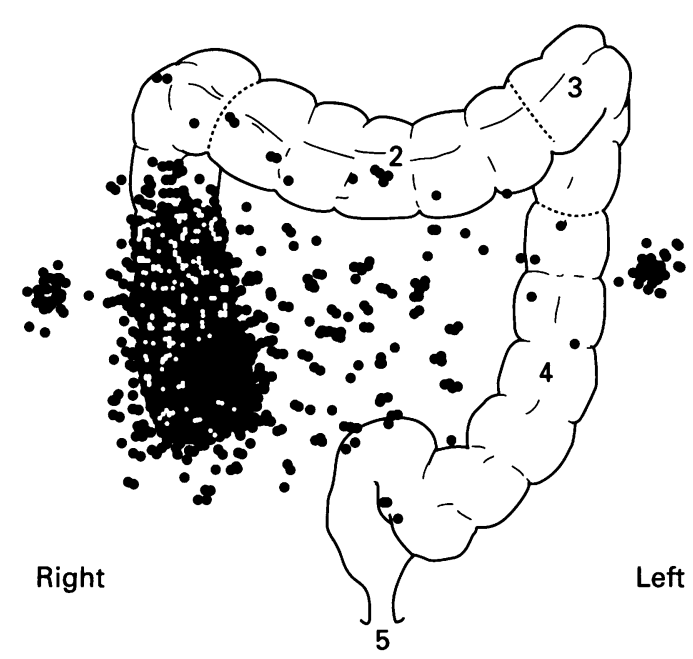

Anterior 10.50

Figure 2: Analogue image showing maximum scintigraphic activity in the caecum.

Differentiating distal ileal activity from activity in the more proximal parts of the bowel could be difficult if relying only on a single image because of overlap of small bowel loops. Determining the time of peak activity in the distal ileum, however, became straightforward when using the dynamic image sequence in conjunction with the derived small bowel counts. Two independent observers ( $\mathrm{RH}$ and AN) determined the time of peak activity in the distal ileum and the time of peak activity in the caecum. There was complete agreement in the determination of these times.

The Mann-Whitney $U$ test was used for statistical comparisons of non-parametric data.

\section{Results}

Figures 3-5 summarise the results. The mean (SD) ileocaecal transit of 103 (50) minutes in patients with IBS was significantly faster than that in control subjects (ileocaecal transit 174 (78) minutes, $\mathrm{p}<0.002$ ).

We subdivided the 20 patients with IBS into 16 who had constipation predominant symptoms and four who had diarrhoea predominant symptoms. The mean (SD) ileocaecal transit of 60 (12) minutes in diarrhoea predominant IBS was faster than that in constipation predominant IBS (115 (50) minutes) and the

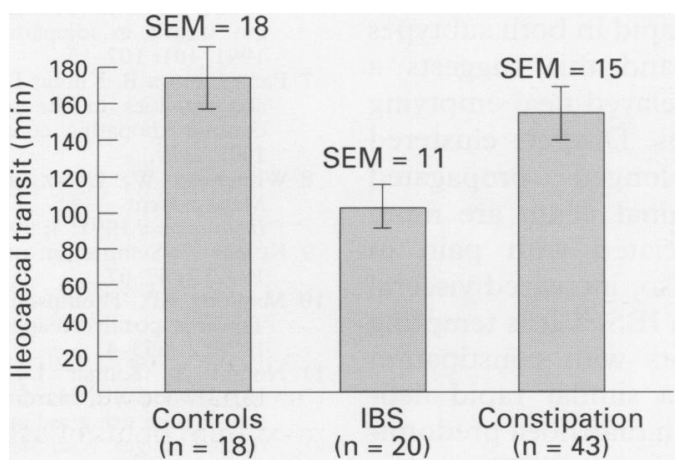

Figure 3: Ileocaecal transit in patients with IBS and chronic idiopathic constipation compared with the control group.

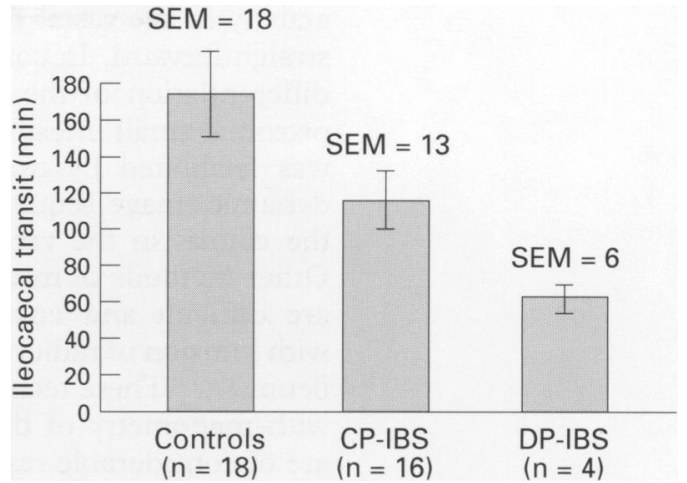

Figure 4: Ileocaecal transit in patients with constipation predominant and diarrhoea predominant IBS. The difference is statistically significant. $C P-I B S=$ constipation predominant IBS, DP-IBS=diarrhoea predominant IBS.

difference was statistically significant $(p<0 \cdot 02)$ (Fig 4).

There were no significant differences in ileocaecal transit between constipated patients and control subjects, or compared with IBS patients. We wondered if any delay in ileocaecal transit in patients with colonic inertia would not be apparent because the constipated group contained patients with anorectal causes of constipation as well as patients with delayed colonic transit. Therefore, the 43 patients with chronic idiopathic constipation were subdivided on the basis of their colonic transit studies into those with normal colonic transit $(n=8)$ and those with delayed colonic transit $(n=35)$. Patients with normal colonic transit had a mean (SD) ileocaecal transit of 128 (97) minutes, which was no different from that in the control subjects. Similarly, patients with delayed colonic transit had a mean (SD) ileocaecal transit of 160 (99) minutes, which was no different from in the control group (Fig 5).

\section{Discussion}

This technique of measuring ileocaecal transit is practical, non-invasive, and conveniently combined with an assessment of colonic transit. Our technique of capsule preparation ${ }^{13}$ is a modification of the method described by Proano et al. ${ }^{12}$ The delivery system was originally reported for the release of drugs in the ileocaecal region. ${ }^{14}$ We have found that identification of the peaks of scintigraphic

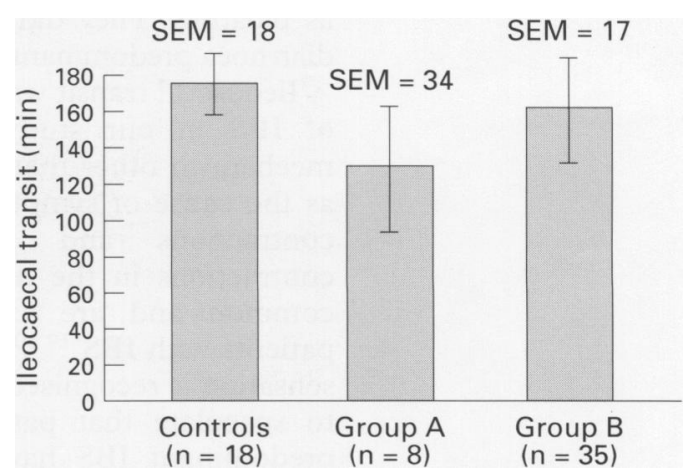

Figure 5: Ileocaecal transit in patients with chronic idiopathic constipation. Group $A=$ constipated patients with normal colonic transit as shown by colonic scintigraphy. Group $B=$ patients with delayed colonic transit (colonic inertia) shown on colonic scintigraphy. 
activity in the distal ileum and the caecum is straightforward. In contrast with expectations, differentiation of the distal ileum from more proximal small intestine was not difficult, and was facilitated by examination of the whole dynamic image sequence in conjunction with the counts in the various regions of interest. Other methods of measuring ileocaecal transit are difficult and entail orocaecal intubation with infusion of radionuclides into the terminal ileum. ${ }^{15-17}$ These techniques can be combined with manometry of the ileocaecal region and are of considerable research value, but are not suitable for wide clinical application. Furthermore, the influence of orocaecal intubation on ileocaecal transit is unknown; it is possible that the presence of a tube traversing the ileocaecal valve changes motility and transit.

Like the scintigraphic ('bran scan') method developed by Trotman and Price, ${ }^{3}$ our technique has the appeal of technical simplicity and is potentially widely applicable. Our delivery system permits reliable release of a bolus of radionuclide in the distal ileum. We can accurately measure time from peak distal ileal activity to peak caecal activity. Therefore, it is not necessary to construct complex time activity curves for ileal emptying or caecal filling. Trotman and Price gave their subjects radiolabelled bran, and high energy meals were given to stimulate ileal emptying of the radionuclide as a bolus. We did not assess ileocaecal transit after a meal stimulus. It remains to be determined whether abnormalities of ileocaecal transit in the fasted state or in response to a meal are clinically relevant.

We found that ileocaecal transit was faster in patients with IBS than in control subjects. Ileocaecal transit was faster in diarrhoea predominant IBS than in constipation predominant IBS, but ileocaecal transit was rapid in both subtypes of IBS compared with controls. The rapid ileocaecal transit in patients with diarrhoea predominant IBS is not surprising, and supports the concept that this subtype of IBS is characterised by rapid small and large bowel transit. ${ }^{18}$ The finding of rapid ileocaecal transit in constipation predominant IBS is unexpected, however, and contrasts with the study by Trotman and Price, which showed prolonged ileocaecal transit in patients with constipation predominant IBS. ${ }^{3}$ They proposed that 'stagnation' of ileal contents accounted for symptoms such as bloating. They did not study patients with diarrhoea predominant symptoms.

Ileocaecal transit was rapid in both subtypes of IBS in our study, and this suggests a mechanism other than delayed ileal emptying as the cause of symptoms. Discrete clustered contractions and prolonged propagated contractions in the terminal ileum are more common and are associated with pain in patients with IBS. ${ }^{19} 20$ Also, increased visceral sensation is recognised in IBS. ${ }^{21}$ It is tempting to speculate that patients with constipation predominant IBS have a similar rapid ileocaecal transit to those with diarrhoea predominant symptoms, but that patients with a sensation of bloating have enhanced sensitivity to ileocaecal dysfunction.
Delayed transit through the proximal colon has been shown in patients with chronic constipation caused by colonic inertia. ${ }^{1122}$ Furthermore, prolonged ambulatory manometry has shown abnormalities of distal ileal motility in these patients. ${ }^{7}$ It seemed reasonable to postulate that ileocaecal transit is prolonged as part of a panenteric disorder of transit and we anticipated finding delayed ileocaecal transit in our patients with chronic constipation. There was no significant prolongation of ileocaecal transit, however, in constipated patients in our study. The patients were a heterogeneous group with some having slow colonic transit, some having impaired rectal evacuation, and others having a combination of functional abnormalities leading to chronic constipation. Nevertheless, the analysis of our normal colonic transit and delayed colonic transit subgroups of constipated patients did not show any difference in ileocaecal transit. We conclude that ileocaecal transit is unchanged in chronic idiopathic constipation. Furthermore, in cases of colonic inertia, the delayed colonic transit does not seem to inhibit ileocaecal transit.

In summary, we have developed a practical, non-invasive scintigraphic method of quantifying ileocaecal transit that can be combined with an assessment of colonic transit. The technique is suitable for routine clinical use in most nuclear medicine departments. We have shown rapid ileocaecal transit in patients with both constipation predominant and diarrhoea predominant types of IBS, but the exact relation between symptoms of IBS and motor abnormalities of the ileocaecal region remains to be elucidated. We have also shown that ileocaecal transit is unchanged in patients with chronic idiopathic constipation. We advocate the use of this scintigraphic technique for the objective quantification of ileocaecal transit.

1 Phillips SF, Quigley EMM, Kumar D, Kamath PS. Motility of the ileocolonic junction. Gut 1988; 29: 390-406.

2 Phillips SF, Camilleri M. The ileocecal area and the irritable bowel syndrome. Gastroenterol Clin North Am 1991; 20: 297-311.

3 Trotman IF, Price CC. Bloated irritable bowel syndrome defined by dynamic ${ }^{99 \mathrm{~m} T c}$ bran scan. Lancet 1986; ii: $364-6$

4 Cann PA, Read NW, Brown C, Hobson N, Holdsworth $\mathrm{CD}$. Irritable bowel syndrome: relationship of disorders in the transit of a single solid meal to symptom patterns. Gut 1983; 24: 405-11.

5 Jian R, Najean Y, Bernier JJ. Measurement of intestinal progression of a meal and its residues in normal subjects progression of a meal and its residues in normal subjects
and patients with functional diarrhoea by a dual isotope and patients with functional diartho

6 Stivland T, Camilleri M, Vassallo M, Proano M, Rath D, Brown $M$, et al. Scintigraphic measurement of regional gut transit in idiopathic constipation. Gastroenterology 1991; 101: 107-15.

7 Panagamuwa B, Kumar D, Keighley MRB, Ortiz J. Motor abnormalities in the terminal ileum in patients with chronic idiopathic constipation. Gastroenterology 1991; 100: A479.

8 Whitehead W, Corazziari E, Chausade S, Kumar D. Management of constipation. Gastroenterology International 1991; 4: 99-114.

9 Kumar D. Symposium on constipation. Int 7 Colorect Dis 1992; 7: 47-67.

10 Manning AP, Thompson WG, Heaton KW, Morris AF. Towards positive diagnosis of the irritable bowel. BMF 1978; 2: 653-4.

11 Notghi A, Kumar D, Panagamuwa B, Tulley NJ, Hesslewood SR, Harding LK. Measurement of colonic Hesslewood SR, Harding LK. Measurement of colonic transit time using radionuclide imaging: analysis by con-

densed images. Nucl Med Commun 1993; 14: 204-11.
Proano M, Camilleri M, Phillips SF, Brown ML Proano M, Camilleri M, Phillips SF, Brown ML,
Thomforde GM. Transit of solids through the colon: regional quantification in the unprepared bowel. $\mathrm{Am} \mathcal{F}$ Physiol 1990; 258: G856-62. 
13 Hesslewood SR, Panagamuwa B, Kumar D, Smith NB, Notghi A, Harding LK. Development of a dosage form for
measuring colonic transit. $\mathcal{F}$ Pharm Pharmacol 1992; 44: measuring.

14 Hardy JG, Wilson CG, Wood E. Drug delivery to the proximal colon. $\mathcal{f}$ Pharm Pharmacol 1985; 37: 874-7.

15 Spiller RC, Brown ML, Phillips SF, Azpiroz F. Scintigraphic measurements of canine ileocolonic transit: direct and indirect effects of eating. Gastroenterology 1986; 91: 1213-20.

16 Spiller RC, Brown ML, Phillips SF. Emptying of the terminal ileum in intact humans: influence of meal residue and ileal motility. Gastroenterology 1987; 92: 724-9.

17 Hammer J, Camilleri M, Phillips SF, Aggarwal A, Haddad AM. Does the ileocolonic junction differentiate between solids and liquids? Gut 1993; 34: 222-6.
18 Lind CD. Motility disorders in the irritable bowel syndrome. Gastroenterol Clin North Am 1991; 20: 279-95.

19 Kellow JE, Phillips SF. Altered small bowel motility in irritable bowel syndrome is correlated with symptoms. Gastroenterology 1987; 92: 1885-93.

20 Kellow JE, Gill RC, Wingate DL. Prolonged ambulan recordings of small bowel motility demonstrate abnormalities in the irritable bowel syndrome. Gastroenterology 1990; 98: 1208-18.

21 Kellow JE, Phillips SF, Miller LJ, Zinsmeister AR Dysmotility of the small intestine in irritable bowel syndrome. Gut 1988; 29: 1236-43.

22 Krevsky B, Maurer AH, Fisher RS. Patterns of colonic transit in chronic idiopathic constipation. Am $\mathcal{f}$ Gastroenterol 1989; 84: $127-32$. 